\title{
The effect of underfeeding on the physiological response to food ingestion in normal weight women
}

\author{
By P. I. MANSELL AND I. A. MACDONALD \\ Department of Physiology and Pharmacology, Medical School, Queen's Medical Centre, \\ Nottingham NG7 $2 U H$
}

(Received 20 October 1987 - Accepted 19 February 1988)

1. The thermogenic, cardiovascular and metabolic responses to the ingestion of a $30 \mathrm{~kJ} / \mathrm{kg}$ body-weight test meal were studied in six normal weight, female subjects before and after a $7 \mathrm{~d}$ period of underfeeding at $60 \mathrm{~kJ} /$ $\mathrm{kg}$ ideal body-weight per $\mathrm{d}$.

2. With underfeeding there were decreases in body-weight, plasma insulin and 3,5,3'-triiodothyronine concentrations, resting metabolic rate and respiratory exchange ratio, with increased blood ketone levels. Baseline 'arterialized' venous plasma noradrenaline and adrenaline concentrations were not affected by underfeeding.

3. Ingestion of the test meal caused similar increases in heart rate and calf blood flow and changes in blood pressure in the fed and underfed states. There was a greater glycaemic response to the test meal in the underfed state compared with the fed state although the rise in plasma insulin concentration was similar and ketogenesis was suppressed. The increases in metabolic rate and plasma noradrenaline concentrations following the test meal were similar in the fed and underfed states.

4. Although the period of underfeeding in the present study led to considerable metabolic adaptation, and some alteration in physiological responses to ingestion of a test meal, there was no evidence that there were associated changes in sympathetic nervous system activation.

Food ingestion induces an increase in metabolic rate (MR), termed the thermic effect of food (TEF). The TEF is usually found to exceed the theoretical, minimum biochemical energy requirements for nutrient digestion and utilization (Flatt, 1978). It has been proposed that the TEF comprises a constant, 'obligatory' component equivalent to the minimum energy cost of nutrient storage plus an additional, variable, 'facultative' component (Acheson et al. 1984). A potential contribution to the facultative component of the TEF derives from the thermogenic effect of sympathetic nervous system (SNS) activation which appears to occur with food and, in particular, carbohydrate ingestion (Welle et al. 1981). Using the glucose clamp technique, the increase in MR, expressed as a function of glucose storage, is approximately halved by previous $\beta$-adrenoceptor blockade; the final level is very similar to the theoretical energy cost of glycogen synthesis (Acheson et al. 1983). Alterations in SNS activity may also contribute to the tachycardia (Welle et al. 1981) and other changes in cardiovascular variables associated with food ingestion.

There is evidence that underfeeding causes a reduction in SNS activity (Landsberg \& Young, 1985). The present study was designed to investigate the degree to which the thermogenic, cardiovascular and metabolic effects of food ingestion are modified by previous underfeeding in normal subjects.

\section{METHODS}

Six healthy, non-obese female subjects were recruited for the study. None was taking any medication other than the contraceptive pill. The ages and physical characteristics of the subjects are given in Table 1. All subjects gave written, informed consent to the study which was approved by the University of Nottingham Medical School Ethical Committee.

Subjects were studied either whilst on their customary diet or following 1 week of underfeeding at $60 \mathrm{~kJ} / \mathrm{kg}$ ideal body-weight per $\mathrm{d}$ (assessed at the mid-point of the 
Table 1. Details of female subjects' ages and physical characteristics

\begin{tabular}{lcc}
\hline & Range & Mean \\
\hline Age (years) & $20-44$ & $26 \cdot 5$ \\
Initial wt $(\mathrm{kg})$ & $51 \cdot 4-70 \cdot 2$ & $63 \cdot 2$ \\
Height $(\mathrm{m})$ & $1 \cdot 59-1 \cdot 77$ & $1 \cdot 69$ \\
Body mass index $\left(\mathrm{kg} / \mathrm{m}^{2}\right)^{*}$ & $20 \cdot 3-24 \cdot 8$ & $22 \cdot 0$ \\
\hline
\end{tabular}

*Weight $(\mathrm{kg}) /$ height $(\mathrm{m})^{2}$.

Metropolitan Life Insurance Company (1959) tables). Details of the composition of each individual's customary food intake were not obtained although the subjects were estimated to be consuming 130 to $160 \mathrm{~kJ} / \mathrm{kg}$ ideal body-weight per $\mathrm{d}$. The diets, supervised by our research dietitian, were designed as an energy-restricted modification of each subject's customary intake, containing ( $\%$ by energy) protein 15-20, carbohydrate $50-60$ and fat $30-40$. Studies were not performed in the week preceding and during the menstrual period to minimize the effect of physiological changes which may occur in some women due to premenstrual sodium and water retention. The study was designed before the influence of phase of the menstrual cycle on metabolic rate was reported (Webb, 1986). Four of the subjects were studied initially in the underfed state and at least 2 weeks elapsed before they were restudied on a normal diet to allow any changes induced by underfeeding to be resolved.

Studies took place in the morning in a temperature-controlled room $\left(30^{\circ}\right)$ with the subjects in the postabsorptive state and wearing shorts and a T-shirt only. Subjects rested supine for at least $30 \mathrm{~min}$ before baseline measurements were made for a further $30 \mathrm{~min}$. Subjects then consumed, as rapidly as possible, and always within $7 \mathrm{~min}$, a standard test meal prewarmed to $37^{\circ}$. The test meal, given at $30 \mathrm{~kJ} / \mathrm{kg}$ body-weight, consisted of a liquid formula preparation (Fortisip plus; Cow and Gate) containing ( $\%$ by energy) 48 carbohydrate, 39 fat and 13 protein (manufacturer's values). Measurements continued for $80 \mathrm{~min}$ after food ingestion.

Continuous recordings of oxygen consumption and carbon dioxide production using a ventilated hood system (Fellows \& Macdonald, 1985) allowed minute-to-minute computation of the respiratory exchange ratio (RER) and of the MR (Weir, 1949). Mean values of MR and RER were calculated for successive 5 min periods for statistical analysis and graphical representation. Heart rate was recorded from an electrocardiogram and averaged over 1 min time-intervals, and brachial arterial blood pressure was measured by auscultation using a mercury sphygmomanometer taking Korotkoff phase $\mathrm{V}$ as the diastolic pressure. Right calf blood flow was determined by venous occlusion plethysmography using a mercury-in-rubber strain gauge (Whitney, 1953).

For blood sampling, a cannula was inserted retrogradely into a superficial vein on the dorsum of the left hand and was kept patent with a slow running infusion (approximately $1 \mathrm{ml} / \mathrm{min}$ ) of saline (154 mmol sodium chloride/l). The hand rested in a box containing air heated to $50-60^{\circ}$. For those substances so far tested, concentrations in 'arterialized' venous blood obtained using this technique approximate to true arterial levels (McGuire et al. 1976). Blood glucose concentration was measured immediately using a Yellow Springs analyser, whilst a portion of blood was deproteinized in perchloric acid, the supernatant fraction being stored at $-20^{\circ}$ for later analysis of lactate, glycerol and $\beta$-hydroxybutyrate (BOHB) levels (Lloyd et al. 1978). The remainder of the blood sample was centrifuged at $4^{\circ}$ and the plasma separated immediately. Plasma $(3 \mathrm{ml})$ was mixed with $100 \mu 1$ EGTA- 
glutathione and stored at $-80^{\circ}$ for determination of noradrenaline and adrenaline concentrations using high-performance liquid chromatography, with electrochemical detection (Macdonald \& Lake, 1985). Additional plasma was stored at $-20^{\circ}$ for subsequent assay of insulin by an in-house double antibody method and of free thyroxine $\left(\mathrm{T}_{4}\right)$ and free 3,5,3'-triiodothyronine $\left(\mathrm{T}_{3}\right)$ concentrations.

Changes in body-weight and thyroid function with underfeeding were assessed using the paired $t$ test. Statistical analysis of the remainder of the values was performed by two-way analysis of variance (ANOVA) with repeated measures provided by the statistical package BMDP. In deriving $P$ values, a Huynh-Feldt correction factor was applied to adjust the number of degrees of freedom. For each variable, analysis was initially undertaken entirely on the baseline values, obtained before ingestion of the test meal, to detect any differences between the normally fed and underfed states. For each subject, mean baseline values were then subtracted from the subsequent values to calculate the magnitude of various physiological responses to the test meal and the latter values were further analysed by ANOVA. Where the $F$ test in ANOVA indicated a significant treatment $\times$ time interaction, the degree of significance was further determined by a $t$ test on the difference between group means at individual time points. In the absence of a treatment $\times$ time interaction, treatment or time effects alone were ascertained. Values are presented as means with their standard errors. The standard errors and indicators of statistical significance on values obtained after ingestion of the test meal refer to the changes from baseline, and not to the original values.

\section{RESULTS}

\section{Weight and thyroid function}

Weight loss with underfeeding ranged from 1.2 to $3.4 \mathrm{~kg}$ (mean 1.95 (SE 0.39 ) $\mathrm{kg}, P<0.01$ ). Plasma free $\mathrm{T}_{4}$ concentration was not significantly different in the underfed state (12.57 (SE 1.15) pmol/1) compared with the fed state (11.42 (SE 0.62) pmol/l). Plasma free $T_{3}$ concentration fell from 5.37 (SE 0.25) pmol/1 to 4.38 (SE 0.38) pmol/1 with underfeeding $(P<0.01)$.

\section{Heart rate, arterial blood pressure and calf blood flow}

Underfeeding induced no statistically significant changes in resting heart rate, systolic or diastolic blood pressures or calf blood flow (Fig. 1). Following ingestion of the test meal, heart rate rose above baseline by approximately 10 beats $/ \mathrm{min}$ in both the fed and underfed states (time effect $F=49 \cdot 23, f_{1} / f_{2}=3 / 15, P<0 \cdot 001$, ANOVA). There were no significant changes in systolic or diastolic blood pressures. Calf blood flow rose following ingestion of the test meal (time effect, $F=155.84, f_{1} / f_{2}=3 / 15, P<0.001$, ANOVA) with maximum increases of 18.7 and $14.6 \mathrm{ml} / 1$ per min in the fed and underfed states respectively, there being no significant difference between these values. The increase in calf blood flow following ingestion of the test meal was due to vasodilatation, manifest by a fall in calf vascular resistance, rather than to a rise in blood pressure.

\section{Blood glycerol, lactate, $B O H B$ and plasma insulin concentrations}

Fasting blood glucose concentration was not significantly reduced by underfeeding (Fig. 2). Following the test meal, there was a greater glycaemic response in the underfed state compared with the fed state (treatment-time effect, $F=9.32, f_{1} / f_{2}=3 / 15, P<0.01$, ANOVA) with maximum increases in blood glucose of 2.60 and $1.22 \mathrm{mmol} / 1$ respectively. Blood glycerol concentration was similar in the fed and underfed states both before and after ingestion of the test meal. Baseline blood lactate concentration was not significantly altered by underfeeding. There was a rise in blood lactate following the test meal (time 

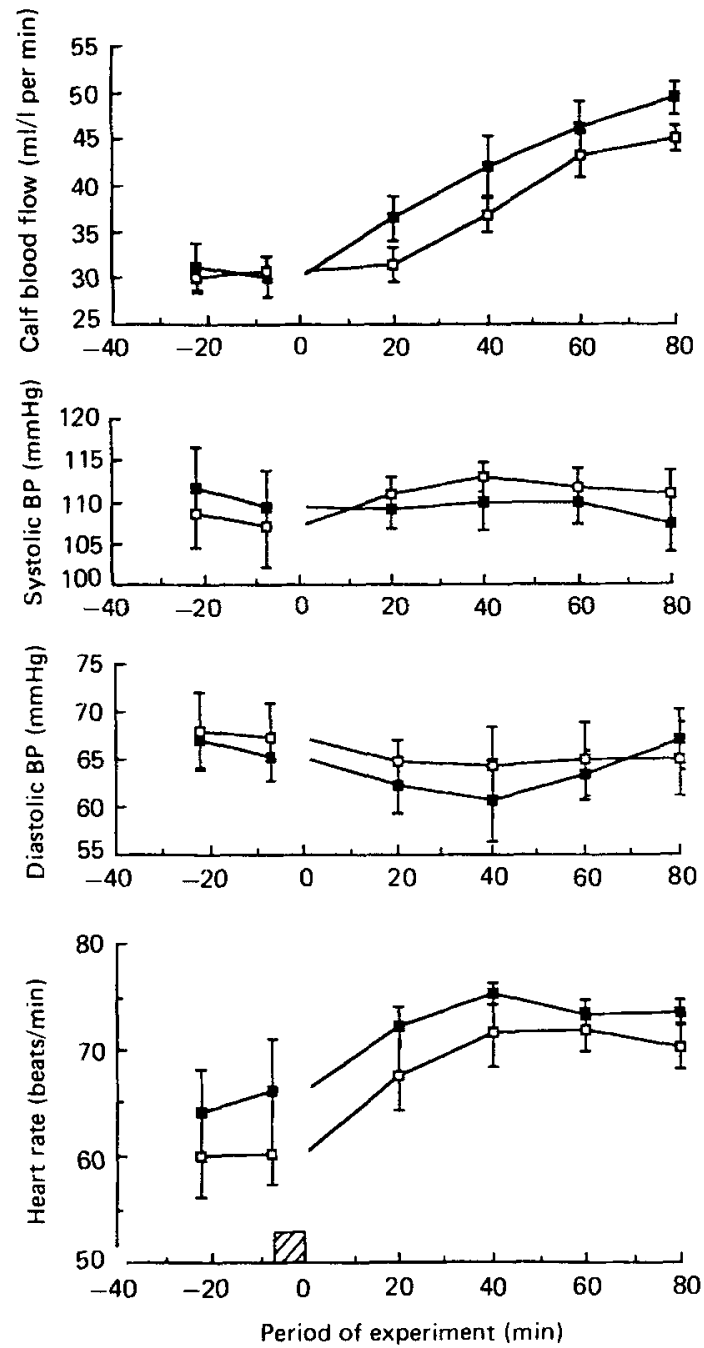

Fig. 1. Mean heart rate, diastolic and systolic blood pressures (BP) and calf blood flow in the fed state (D) and underfed state ( $\square$ ), before and after food ingestion. (Q) Time-period of food ingestion. Values are means with their standard errors represented by vertical bars. Standard errors refer to baseline values and to the changes from baseline induced by ingestion of the test meal. There were no effects of underfeeding on baseline values for any variable. Following ingestion of the test meal, there were increases in both heart rate $(P<0.001$, ANOVA) and calf blood flow $(P<0.001$, ANOVA). Responses to ingestion of the test meal were independent of the previous state of feeding.

effect, $F=7.33, f_{1} / f_{2}=3 / 15, P<0.01$, ANOVA), with maximum increases of 0.41 and $0.34 \mathrm{mmol} / 1$ in the fed and underfed states respectively. The rise in blood lactate concentration was independent of the previous state of feeding. Baseline blood BOHB concentration was increased by underfeeding from 0.055 to $0.404 \mathrm{mmol} / \mathrm{l}$ (treatment effect, $F=6.86, f_{1} / f_{2}=1 / 5, P<0.05$, ANOVA). Following ingestion of the test meal, blood BOHB concentration fell (time effect, $F=13.76, f_{1} / f_{2}=3 / 15, P<0.01$, ANOVA), the fall being to similar low levels in both the fed and underfed states. Baseline plasma insulin concentration was reduced from 12.5 to $7.3 \mathrm{mU} / 1$ with underfeeding $(P<0.05$, paired $t$ 

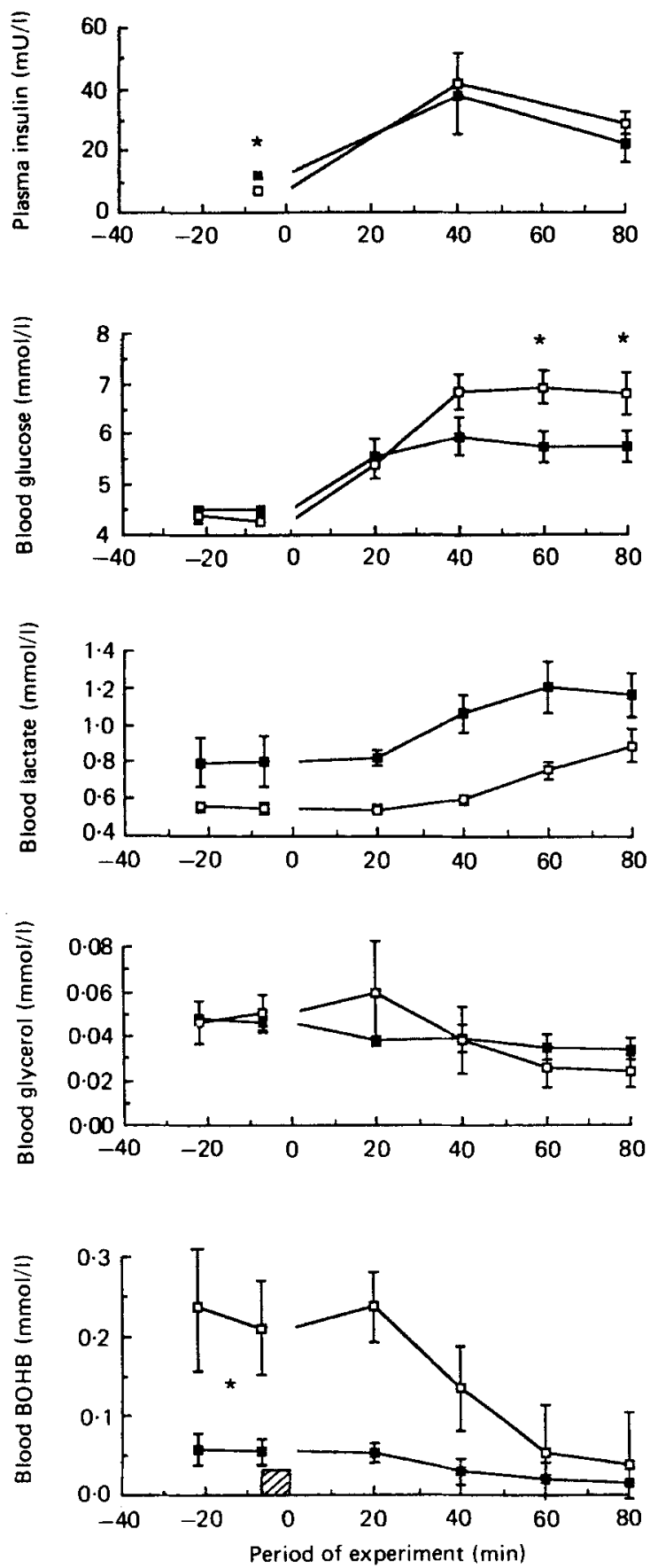

Fig. 2. Mean values for blood $\beta$-hydroxybutyrate (BOHB), glycerol, lactate, glucose and plasma insulin concentrations in the fed state $(\square)$ and underfed state $(\square)$ before and after food ingestion. ( $\square$ ) Timeperiod of food ingestion. Values are means with their standard errors represented by vertical bars. Standard errors refer to the baseline values and to the changes from baseline induced by ingestion of the test meal. Statistically significant differences between the fed and underfed states in both baseline values and in responses to ingestion of the test meal are shown $\left({ }^{*} P<0.05\right)$. 

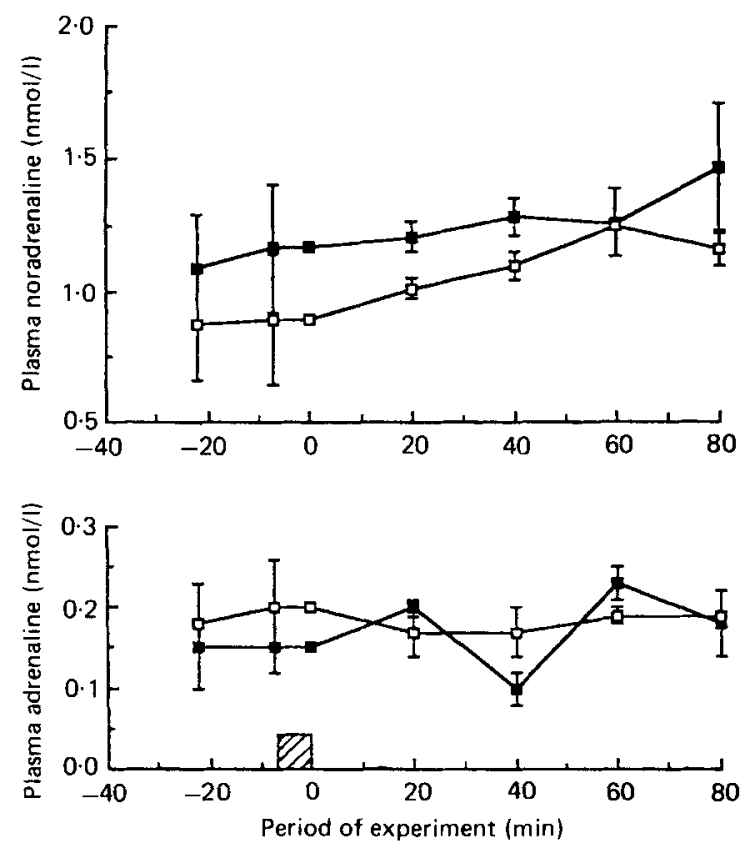

Fig. 3. Mean values for plasma noradrenaline and adrenaline concentrations in the fed state ( $\square$ ) and underfed state $(\square)$ before and after food ingestion. ( $\square$ ) Time-period of food ingestion. Values are means with their standard errors represented by vertical bars. Standard errors are given for the baseline values and the changes from baseline induced by ingestion of the test meal. There was no significant effect of underfeeding on baseline values or responses to ingestion of the test meal for either variable. Following ingestion of the test meal, there was a rise in plasma noradrenaline concentration in both fed and underfed states $(P<0.01$, ANOVA).

test) but, following ingestion of the test meal, plasma insulin rose to similar levels in both fed and underfed states.

\section{Plasma adrenaline and noradrenaline concentrations}

Baseline plasma noradrenaline concentration was $1.12 \mathrm{nmol} / 1$ in the normally fed state and $0.88 \mathrm{nmol} / 1$ in the underfed state, with no significant difference (Fig. 3). There was an overall increase in plasma noradrenaline levels following ingestion of the test meal $(F=$ $22.00, f_{1} / f_{2}=1 / 4, P<0.01$, ANOVA), the magnitude of this increase being the same in the fed and underfed states. Baseline plasma adrenaline concentration was unaltered by underfeeding and did not change following ingestion of the test meal.

\section{$M R$ and $R E R$}

With underfeeding, resting MR was reduced from 4.21 to $3.91 \mathrm{~kJ} / \mathrm{min}$ (treatment effect, $F=10.38, f_{1} / f_{2}=1 / 5, P<0.05$, ANOVA) (Fig. 4). The mean rise in MR above baseline in the $80 \mathrm{~min}$ of measurement following food ingestion (the TEF) was $0.30 \mathrm{~kJ} / \mathrm{min}$ in the fed state and $0.32 \mathrm{~kJ} / \mathrm{min}$ in the underfed state with no significant difference. For each subject, the overall increase in plasma noradrenaline concentration following ingestion of the test meal was calculated as the area under the curve above baseline of plasma noradrenaline concentration. Using linear regression analysis, there was no correlation between this area under the plasma noradrenaline curve and the TEF in individual subjects, either in the fed or underfed states or with the two sets of values combined. Mean baseline RER fell from 0.81 in the fed state to 0.73 in the underfed state (treatment effect, $F=16.05$, 

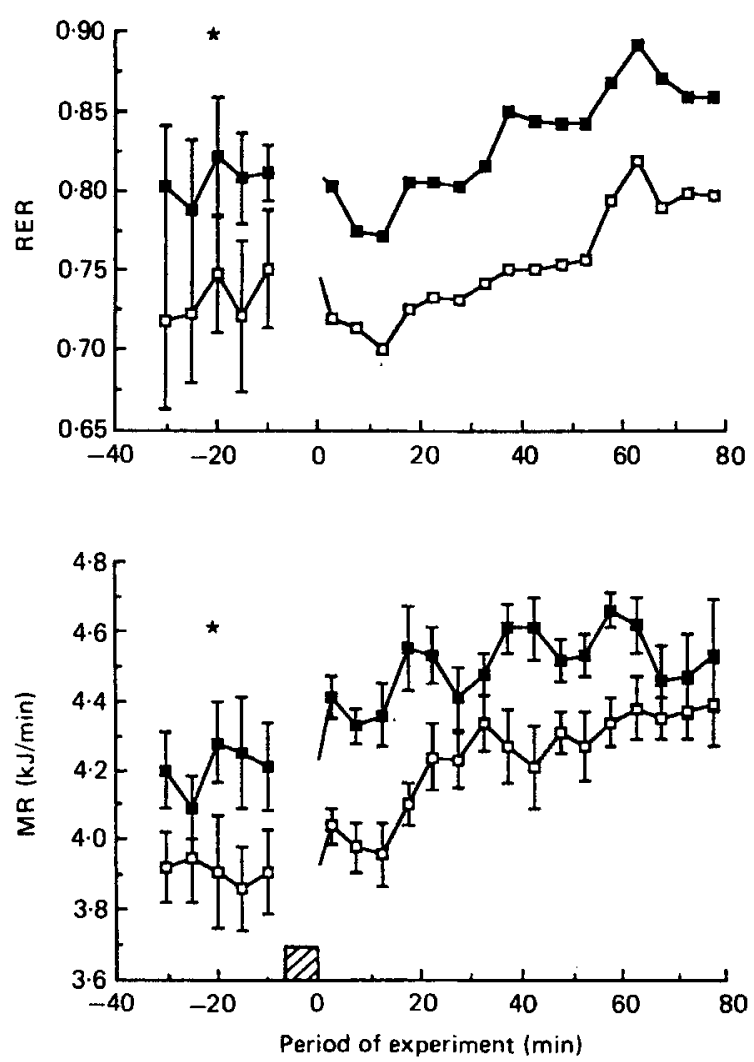

Fig. 4. Metabolic rate (MR) and respiratory exchange ratio (RER) in the fed state ( $\square$ ) and underfed state $(\square)$ before and after food ingestion. ( $\square$ ) Time-period of food ingestion. Values are means with their standard errors represented by vertical bars. The standard errors are given for the baseline values and on the change in MR from baseline induced by ingestion of the test meal. Statistically significant differences between the fed and underfed states are shown $\left({ }^{*} P<0.05\right)$. Changes in RER following ingestion of the test meal were not analysed statistically.

$\left.f_{1} / f_{2}=1 / 5, P<0.01\right)$. RER rose following food ingestion, there being a parallel rise in the fed and underfed states with values for the latter remaining lower.

\section{DISCUSSION}

The period of underfeeding was accompanied by weight loss and by considerable changes in basal metabolism. There was a reduction in RMR with underfeeding as reported previously (Jung et al. 1980) and a fall in RER consistent with a shift towards fat as the major fuel source. Blood ketone (BOHB) concentrations increased and there was a fall in plasma insulin concentration. Plasma $T_{3}$ was reduced by underfeeding with plasma $T_{4}$ remaining unaltered, as observed previously with restriction of energy intake (Jung et al. 1980). It appears most likely that any changes in physiological responses in the present study were due to overall reductions in food intake but the possibility cannot be excluded that there may have been some effect resulting from possible alterations in dietary composition.

There was no significant change in resting heart rate with underfeeding. A fall in both systolic and diastolic arterial blood pressures with energy restriction has been described in 
normotensive, obese subjects. This was reported to occur in association with a fall in SNS activity as assessed by a reduction in the $24 \mathrm{~h}$ urinary excretion of catecholamine metabolites (Jung et al. 1979). In the present study there was no change in blood pressure with underfeeding although our subjects had considerably lower initial values and underwent a shorter, less intensive period of energy restriction than the obese subjects described previously (Jung et al. 1979).

The similar changes in heart rate, blood pressure and calf blood flow following food ingestion in the fed and underfed states are consistent with similar changes in SNS activity in mediating cardiovascular responses in the two states. Although our study was not placebo controlled, it is highly probable that ingestion of the test meal was responsible for the subsequent considerable rise in calf blood flow as in other, similar studies, little change in blood flow occurred without intervention (Fellows et al. 1985). The present results do not provide any indications as to the mechanism of this post-prandial increase in calf blood flow, except that it is manifestly not due to an increase in circulating adrenaline levels. An increase in splanchnic blood flow of $50-100 \%$ following food ingestion has been reported (Qamar \& Read, 1985). Together with the increase in calf blood flow this would imply a considerable increase in cardiac output post-prandially rather than a diversion of blood flow from the limbs to the viscera.

Underfeeding is accompanied by a decrease in glycogen stores and an increase in the use of fat as an energy source. The increased blood BOHB concentration seen with underfeeding is consistent with enhanced hepatic ketone body formation from fatty acids, although non-esterified fatty acid levels were not measured. The rate of glycerol production would be enhanced by increased lipolysis but there was no change in blood glycerol concentration with underfeeding in the present study. This may have been due to an increased rate of utilization of glycerol for gluconeogenesis. Blood lactate concentration depends on a balance between peripheral glycolysis and glycogenolysis, and hepatic utilization of lactate for gluconeogenesis. There was no significant change in blood lactate concentration with underfeeding, perhaps due to these competing influences. Underfeeding caused no significant change in baseline blood glucose but the glycaemic response to the test meal was approximately doubled. It is well-recognized that reducing carbohydrate intake leads to glucose intolerance (De Fronzo et al. 1978).

Baseline plasma insulin concentration was reduced by underfeeding. Compared with the fed state, consumption of the test meal after underfeeding led to a similar rise in plasma insulin concentration. This parallel rise was accompanied by relative hyperglycaemia in the underfed state which implies a degree of resistance to the hypoglycaemic action of insulin. There was, however, also a contribution from insulin undersecretion as higher blood glucose levels would normally have induced enhanced insulin secretion, which was not observed. The resistance to the action of insulin in stimulating glucose utilization in the underfed state does not extend to the effect of insulin in suppressing hepatic ketogenesis. This is apparent from the sharp decline in blood BOHB concentration following ingestion of the test meal in the underfed state. This implies that underfeeding exerts a differential effect on the control of glycaemia and ketonaemia by insulin.

Underfeeding appears to be accompanied by a fall in SNS activity (Jung et al. 1979; Schwartz et al. 1983). This may mediate some of the physiological changes seen in undernutrition, such as the falls in heart rate, blood pressure and MR (Jung et al. 1980; Landsberg \& Young, 1985). In the forearm vascular bed, both uptake and local release of noradrenaline occur, thereby influencing antecubital venous plasma concentrations (Chang et al. 1986). Therefore arterial or arterialized venous plasma noradrenaline concentrations may provide a more direct index of overall SNS activity. However, the steady state concentration is dependent not only on the rate of release of noradrenaline from 
sympathetic nerve endings but also on the rate of clearance which itself may be reduced by underfeeding (Esler, 1982). In the present study, although there was a trend towards lower arterialized venous plasma noradrenaline concentrations with underfeeding, there was no statistically significant change, despite marked metabolic responses to this period of food restriction. However, a lack of indirect evidence of this type does not preclude a fall in SNS activity with underfeeding, at least in that part of the SNS involved in thermogenesis.

Stock (1980) found no alteration in the thermic effect of a $1.67 \mathrm{~kJ}$ meal after a $36 \mathrm{~h}$ complete fast in normal subjects. In a study of young women who were not obese (98-118\% ideal body-weight) but who considered they had a weight problem, Morgan (1984) found that the TEF was not affected by $14 \mathrm{~d}$ underfeeding at $4.0 \mathrm{MJ} / \mathrm{d}$ but was reduced by a subsequent $4 \mathrm{~d}$ period of dieting at $1.7 \mathrm{MJ} / \mathrm{d}$. There is evidence that, with a meal of $1670 \mathrm{~kJ}(400 \mathrm{kcal})$ or more, the TEF is reduced in the obese compared with normal subjects (Schwartz et al. 1983; Swaminathan et al. 1985). This may predispose to weight gain and may be due to genetic differences. With underfeeding and weight reduction in the obese there appears to be a further decrease in the TEF when glucose is used (Schutz et al. 1984), although not when a mixed meal is given (Shetty et al. 1981).

In the present study there was no reduction in the MR response to food ingestion after a previous period of underfeeding. Over the $80 \mathrm{~min}$ of measurement, the mean increase in MR in the underfed state was $0.32 \mathrm{~kJ} / \mathrm{min}$ compared with $0.30 \mathrm{~kJ} / \mathrm{min}$ in the fed state. There was, however, a degree of glucose intolerance in the underfed state, implying a diminished rate of glucose storage which might, if anything, have been expected to lead to a reduction in the TEF. The TEF is incomplete by $80 \mathrm{~min}$ and its time course might be affected by changes in previous nutritional state, thereby potentially influencing the MR response observed; this is a problem which besets all similar studies. In particular, a biphasic sympatho-adrenomedullary response to glucose ingestion has recently been reported, the second phase of which begins at 3-4 $\mathrm{h}$ after ingestion (Astrup et al. 1986). In the $80 \mathrm{~min}$ of study, any effect of underfeeding on the physiological responses associated with this second phase of catecholamine release would therefore have been missed.

The assimilation of food, and in particular carbohydrate ingestion or infusion, appears to be associated with an increase in SNS activity as judged by increases in plasma noradrenaline concentration, heart rate and MR (Acheson et al. 1984; Rowe et al. 1981; Welle et al. 1981). In addition, the thermic effect of infused glucose is reduced by previous $\beta$-adrenoceptor blockade, consistent with a sympathetically mediated component (Acheson et al. 1983). In the present study, there was a moderate rise in plasma noradrenaline concentration following ingestion of the test meal. However there was no correlation between the TEF and the rise in plasma noradrenaline in individuals, in accord with the observations in obese subjects made by Welle \& Campbell (1983). More recently, studies involving the infusion of radiolabelled noradrenaline have shown a correlation between the TEF and the rate of appearance of noradrenaline in the circulation (Schwartz et al. 1987).

As underfeeding appears to lead to a reduction in SNS activity, it is possible that the degree of activation of the SNS and the associated physiological responses induced by ingestion of a meal might depend on the previous level of nutritional intake. In the present study, there was no evidence that this was the case. The increase in plasma noradrenaline concentration induced by ingestion of the test meal was similar in the fed and underfed states. In addition, underfeeding did not significantly modify the cardiovascular and thermogenic responses to ingestion of the test meal.

The study was supported by a project grant from the Wellcome Trust. The authors thank Jo Rawlings, research dietitian, who supervised the underfeeding. 


\section{REFERENCES}

Acheson, K., Jequier, E. \& Wahren, J. (1983). Journal of Clinical Investigation 72, 981-986.

Acheson, K., Ravussin, E., Wahren, J. \& Jequier, E. (1984). Journal of Clinical Investigation 74, 1572-1580.

Astrup, A., Bulow, J., Christensen, N. J., Madsen, J. \& Quaade, F. (1986). American Journal of Physiology 250, E226-E229.

Chang, P. C., van der Krogt, J. A., Vermeij, P. \& van Brummelen, P. (1986). Hypertension 8, 801-809.

De Fronzo, R. A., Soman, V., Sherwin, R. W., Hendler, R. \& Felig, P. (1978). Journal of Clinical Investigation 62, 204-213.

Esler, M. (1982). Clinical Science 62, 247-254.

Fellows, I. W., Bennett, T. \& Macdonald, I. A. (1985). Clinical Science 69, 215-222.

Fellows, I. W. \& Macdonald, I. A. (1985). Clinical Physics and Physiological Measurement 6, 349-355.

Flatt, J. P. (1978). In Recent Advances in Obesity Research, pp. 211-218 [G. A. Bray, editor]. London: Newman.

Jung, R. T., Shetty, P. S., Barrand, M., Callingham, B. A. \& James, W. P. T. (1979). British Medical Journal i, 12-13.

Jung, R. T., Shetty, P. S. \& James, W. P. T. (1980). Clinical Science 58, 183-191.

Landsberg, L. \& Young, J. B. (1985). In Neuroendocrine Perspectives, vol. 4, pp. 191-218 [E. E. Miller, R. M. Macleod and L. A. Frohman, editors]. New York: Elsevier Science Publishers.

Lloyd, B., Burin, J., Smythe, P. \& Alberti, K. G. M. M. (1978). Clinical Chemistry 24, 1724-1729.

Macdonald, I. A. \& Lake, D. M. (1985). Journal of Neuroscience Methods 13, 239-248.

McGuire, F., Heldman, J., Tobin, J., Andres, R. \& Berman, M. (1976). Journal of Applied Physiology 41, $565-573$.

Metropolitan Life Insurance Company (1959). Statistical Bulletin 40, 1.

Morgan, J. B. (1984). International Journal of Obesity 8, 629-640.

Qamar, M. F. \& Read, A. E. (1985). Quarterly Journal of Medicine 56, no. 220, 417-419.

Rowe, J. W., Young, J. B., Minaker, K. L., Stevens, A. L., Pallotta, J. \& Landsberg, L. (1981). Diabetes 30, 219-225.

Schutz, Y., Golay, A., Felber, J.-P. \& Jequier, E. (1984). American Journal of Clinical Nutrition 39, $380-387$.

Schwartz, R. S., Halter, J. B. \& Bierman, E. L. (1983). Metabolism 32, 114-117.

Schwartz, R. S., Jaeger, L. F., Silberstein, S. \& Veith, R. C. (1987). International Journal of Obesity 11, 141-149.

Shetty, P. S., Jung, R. T., James, W. P. T., Barrange, M. A. \& Callingham, B. A. (1981). Clinical Science 60 , 519-525.

Stock, M. J. (1980). European Journal of Applied Physiology 43, 35-40.

Swaminathan, R., King, R. F. G. J., Holmfield, J., Swieck, R. A., Baker, M. \& Wales, J. K. (1985). American Journal of Clinical Nutrition 42, 177-181.

Webb, P. (1986). American Journal of Clinical Nutrition 44, 614-619.

Weir, J. B. de V. (1949). Journal of Physiology 109, 1-9.

Welle, S. \& Campbell, R. G. (1983). American Journal of Clinical Nutrition 37, 87-92.

Welle, S., Lilavivat, U. \& Campbell, R. G. (1981). Metabolism 30, 953-958.

Whitney, R. J. (1953). Journal of Physiology 121, 1-27. 